\title{
Efficient operators for studying higher partial waves
}

\author{
Jia-jun Wu ${ }^{1,2, \star}$, Waseem Kamleh ${ }^{1}$, Derek B. Leinweber ${ }^{1}$, Gerrit Schierholz ${ }^{3}$, Ross D. Young ${ }^{1}$, and \\ James $M$. Zanotti ${ }^{1}$ \\ ${ }^{1}$ Special Research Centre for the Subatomic Structure of Matter (CSSM), Department of Physics, University \\ of Adelaide, Adelaide, South Australia 5005, Australia \\ ${ }^{2}$ HISKP (Theory) and BCTP, University of Bonn, Germany \\ ${ }^{3}$ Deutsches Elektronen-Synchrotron DESY, 22603 Hamburg, Germany
}

\begin{abstract}
An extended multi-hadron operator is developed to extract the spectra of irreducible representations in the finite volume. The irreducible representations of the cubic group are projected using a coordinate-space operator. The correlation function of this operator is computationally efficient to extract lattice spectra. In particular, this new formulation only requires propagator inversions from two distinct locations, at fixed physical separation. We perform a proof-of-principle study on a $24^{3} \times 48$ lattice volume with $m_{\pi} \approx 900 \mathrm{MeV}$ by isolating the spectra of $A_{1}^{+}, E^{+}$and $T_{2}^{+}$of the $\pi \pi$ system with isospin-2 in the rest frame.
\end{abstract}

\section{Introduction}

The numerical simulation of quark and gluon fields on a finite lattice enables a study of the hadron spectrum and strong interactions of QCD via first principles. Recently, lattice QCD calculations of the hadron spectrum have achieved tremendous progress $[1,2]$. High partial wave states are an important topic in hadron physics, yet it is still a challenge to study these states on the lattice. The relevant symmetry group on a 4-dimensional lattice is reduced from the $\mathrm{SO}(3)$ group of the infinite volume to the cubic group of a finite volume. As a result, the irreducible representation of the cubic group has a nonzero overlap with infinitely many partial wave states which are the basis states of irreducible representations of the $\mathrm{SO}(3)$ group. Therefore the energy eigenvalues of each irreducible representation will receive the contributions from the phase shifts in an infinite number of partial waves $[3,4]$. There have been a number of studies outlining the relations between discrete energy levels and phase shifts, including the mixing of high partial waves, e.g. [3-8]. For these reasons, the spectra of irreducible representations of the cubic group are key to studying high partial wave states in a lattice calculation.

The major difficulty lies in the extraction of the spectrum of each unique irreducible representation. In this proceedings, we introduce a novel technique for determining the spectra of various irreducible representations of a 2-body system. The method relies upon constructing an operator that corresponds to a "shell" in coordinate space, which has similarities to the "cube" source employed in [9], where the two body operator $\psi$ is the product of two single particle operators separated by a distance $\vec{\delta}$.

${ }^{\star}$ Speaker, e-mail: jiajun.wu@ adelaide.edu.au 
As we will show, by summing over lattice rotations, $\hat{R}$, we construct an operator, $\Psi_{\Gamma}=\sum_{\hat{R}} C(\hat{R}) \psi_{\hat{R}}$, which projects onto a given irreducible representation, $\Gamma$. The correlation function of the operator $\Psi_{\Gamma}$ will generate a clean spectrum of the corresponding irreducible representation $\Gamma$. As an exploratory exercise, we study the isospin- $2 \pi \pi$ system, for which several alternative methods have already been explored [10-12]. We demonstrate that we are able to successfully determine energy levels of the $A_{1}^{+}$, $E^{+}$and $T_{2}^{+}$representations.

\section{Formalism}

\section{$2.1 O_{h}$ group}

In the centre-of-mass (CM) frame in a finite volume, the basic symmetry group of the cubic lattice for integer spin particles is the cubic group, $O$. The full symmetry group includes space inversions $\hat{\pi}$, therefore, the full group should be the product of the cubic group and spatial inversions, i.e., $O_{h}=$ $O \otimes V_{2}$. There are 48 elements in the $O_{h}$ group, and they fall into ten different conjugacy classes. There are ten irreducible representations of the $O_{h}$ group. The character table of the $O_{h}$ group is shown in Table $1[6]$.

Table 1. Character table of the $O_{h}$ group.

\begin{tabular}{lllllllllll}
\hline$\Gamma /$ Class & $I$ & $8 C_{3}^{\prime}$ & $6 C_{4}$ & $6 C_{4}^{\prime}$ & $3 C_{4}^{2}$ & $\hat{\pi}$ & $8 C_{3}^{\prime} \times \hat{\pi}$ & $6 C_{4} \times \hat{\pi}$ & $6 C_{4}^{\prime} \times \hat{\pi}$ & $3 C_{4}^{2} \times \hat{\pi}$ \\
\hline$A_{1}^{ \pm}$ & 1 & 1 & 1 & 1 & 1 & \pm 1 & \pm 1 & \pm 1 & \pm 1 & \pm 1 \\
$A_{2}^{ \pm}$ & 1 & 1 & -1 & -1 & 1 & \pm 1 & \pm 1 & $\mp 1$ & $\mp 1$ & \pm 1 \\
$E^{ \pm}$ & 2 & -1 & 0 & 0 & 2 & \pm 2 & $\mp 1$ & 0 & 0 & \pm 2 \\
$T_{1}^{ \pm}$ & 3 & 0 & 1 & -1 & -1 & \pm 3 & 0 & \pm 1 & $\mp 1$ & $\mp 1$ \\
$T_{2}^{ \pm}$ & 3 & 0 & -1 & 1 & -1 & \pm 3 & 0 & $\mp 1$ & \pm 1 & $\mp 1$ \\
\hline
\end{tabular}

In the CM frame, full rotational symmetry is described by the $S O(3)$ group, and the familiar basis states denoted by $|l m\rangle$. Here $l$ and $m$ are the usual quantum numbers of total and $z$-component of angular momentum, respectively. However, in the finite volume, angular momentum is no longer a good quantum number since the symmetry reduces to the $O_{h}$ group. The ten irreducible representations of $O_{h}$ in a finite volume are then mixtures of an infinite number of angular momentum states defined in the infinite volume. In Table 2, we list the angular momentum states up to $l=9$ contained in the ten irreducible representations [7].

Table 2. The list of angular momenta, $l<10$, appearing in each irreducible representation, $\Gamma$, of $O_{h}$ [7].

\begin{tabular}{llllll}
\hline$\Gamma$ & $A_{1}^{+}$ & $A_{2}^{+}$ & $E^{+}$ & $T_{1}^{+}$ & $T_{2}^{+}$ \\
$l$ & $(0,4,8, \ldots)$ & $(6, \ldots)$ & $(2,4,6,8, \ldots)$ & $(4,6,8, \ldots)$ & $(2,4,6,8, \ldots)$ \\
\hline$\Gamma$ & $A_{1}^{-}$ & $A_{2}^{-}$ & $E^{-}$ & $T_{1}^{-}$ & $T_{2}^{-}$ \\
$l$ & $(9, \ldots)$ & $(3,7,9, \ldots)$ & $(5,7,9, \ldots)$ & $(1,3,5,7,9, \ldots)$ & $(3,5,7,9, \ldots)$ \\
\hline
\end{tabular}

\subsection{Operator}

The standard lattice operator for the pion is :

$$
\phi(t ; \vec{x}) \equiv \sum_{a} \bar{u}^{a}(t ; \vec{x}) \gamma_{5} d^{a}(t ; \vec{x}),
$$


with a sum over colour indices $a$.

In coordinate space, we construct a composite operator with a separation $\vec{\delta}$ between two pions:

$$
\psi(t ; \vec{x}, \vec{\delta}) \equiv \phi(t ; \vec{x}+\vec{\delta} / 2) \phi(t ; \vec{x}-\vec{\delta} / 2) .
$$

This operator is equivalent to any other that is one of the 48 rotation operators, $\hat{R} \in O_{h}$ group. We then denote the 48 pion-pion operators $\psi_{\hat{R}}$ as:

$$
\psi_{\hat{R}}(t ; \vec{x}, \vec{\delta})=\hat{P}_{\hat{R}} \psi(t ; \vec{x}, \vec{\delta}) \hat{P}_{\hat{R}^{-1}}=\psi\left(t ; \hat{R}^{-1} \vec{x}, \hat{R}^{-1} \vec{\delta}\right)=\phi\left(t ; \hat{R}^{-1}(\vec{x}+\vec{\delta} / 2)\right) \phi\left(t ; \hat{R}^{-1}(\vec{x}-\vec{\delta} / 2)\right) .
$$

In this work, we maximize the symmetry available by avoiding equivalent rotations, i.e., $\hat{R} \vec{\delta} \neq \vec{\delta}$. This simply requires $\delta_{x} \neq \delta_{y} \neq \delta_{z} \neq 0$. While the coordinate position of each pion should be on the lattice grid, i.e, $\hat{R}(\vec{x} \pm \vec{\delta} / 2) \in \mathbb{Z}^{3}$, the origin of the composite operator need not be on a lattice site. One convenient choice is $\vec{\delta}=(1,3,5)$ and $\vec{x}=(1 / 2,1 / 2,1 / 2)$, which keeps the shortest $|\vec{\delta}|$.

By using the above operators, the $\left|\pi^{-}(t, \vec{x}+\vec{\delta} / 2) \pi^{-}(t, \vec{x}+\vec{\delta} / 2)\right\rangle$ system can be generated as $\psi^{\dagger}(t ; \vec{x}, \vec{\delta})|\Omega\rangle$ where $|\Omega\rangle$ is the vacuum state. Correspondingly, 48 different states can be generated according to Eq.(3) through the use of rotation operators. For simplification, we use $\left|\psi_{\hat{R}}^{\dagger}(t ; \vec{x}, \vec{\delta})\right\rangle$ to replace the $\left|\pi^{-}\left(t, \hat{R}^{-1}(\vec{x}+\vec{\delta} / 2)\right) \pi^{-}\left(t, \hat{R}^{-1}(\vec{x}+\vec{\delta} / 2)\right)\right\rangle$ system. These pion-pion systems should obey rotational symmetry as follows:

$$
\hat{P}_{\hat{R}}\left|\psi_{\hat{R}^{\prime}}^{\dagger}(t ; \vec{x}, \vec{\delta})\right\rangle=\sum_{\hat{R}^{\prime \prime} \in O_{h}}\left|\psi_{\hat{R}^{\prime \prime}}^{\dagger}(t ; \vec{x}, \vec{\delta})\right\rangle(\bar{B}(\hat{R}))_{\hat{R}^{\prime \prime}, \hat{R}^{\prime}},
$$

where matrix $(\bar{B}(\hat{R}))_{\hat{R}^{\prime \prime}, \hat{R}^{\prime}}=\delta_{\hat{R} \hat{R}^{\prime}, \hat{R}^{\prime \prime}}$, is an element of the regular representation of the group $O_{h}$. We note that we have introduced the notation where we directly use the 48 rotation operators of the $O_{h}$ group as indices of the matrix $\bar{B}$. Furthermore, here " $\bar{B}$ " refers to the matrix representation of $B$, e.g., the matrix of irreducible representation $\Gamma$ is written as $\bar{\Gamma}$.

The regular representation of the $O_{h}$ group is reducible. Through the similarity transformation matrix, $\bar{S}$, all of these 48 matrices, $\bar{B}(\hat{R})$, can be block diagonalised into a matrix $\bar{A}$. Each nonzero block matrix is in an irreducible representation:

$$
\bar{S}^{-1} \bar{B}(\hat{R}) \bar{S}=1 \bar{A}_{1}^{ \pm}(\hat{R}) \oplus 1 \bar{A}_{2}^{ \pm}(\hat{R}) \oplus 2 \bar{E}^{ \pm}(\hat{R}) \oplus 3 \bar{T}_{1}^{ \pm}(\hat{R}) \oplus 3 \bar{T}_{2}^{ \pm}(\hat{R}) \equiv \bar{A}(\hat{R}) .
$$

The number before the irreducible representation indicates the number of copies of that representation. Therefore, it is convenient to set the indicies of $\bar{A}$ as the group of three numbers, $(i, \Gamma, n)$, where $i$ indicates the $i$-th irreducible representation, $\Gamma$ its name, and $n$ indicates its order. These matrices $\bar{A}$ can be written as:

$$
\bar{A}_{i \Gamma n, i^{\prime} \Gamma^{\prime} n^{\prime}}(\hat{R})=\delta_{i^{\prime} i} \delta_{\Gamma^{\prime} \Gamma} \bar{\Gamma}_{n, n^{\prime}}(\hat{R}),
$$

Because $O_{h}$ is a finite group, matrices $\bar{\Gamma}$ can be chosen as unitary matrices.

As shown in Eq. (4), the matrix $\bar{B}(\hat{R})$ describes the rotations between the 48 states, $\left|\psi_{\hat{R}}\right\rangle$. Similarly, the matrix $\bar{A}$ describes rotations between systems classified in terms of the irreducible representations, $\left|\Psi_{i, \Gamma, n}^{\dagger}\right\rangle$, satisfying:

$$
\hat{P}_{R}\left|\Psi_{i, \Gamma, n}^{\dagger}\right\rangle=\sum_{i^{\prime}, \Gamma^{\prime}, n^{\prime}}\left|\Psi_{i^{\prime}, \Gamma^{\prime}, n^{\prime}}^{\dagger}\right\rangle(\bar{A}(\hat{R}))_{i^{\prime} \Gamma^{\prime} n^{\prime}, i \Gamma n} .
$$

The transformation matrix $\bar{S}$ connects $\left|\psi_{\hat{R}}^{\dagger}\right\rangle$ and $\left|\Psi_{i, \Gamma, n}^{\dagger}\right\rangle$ as follows:

$$
\left|\Psi_{i, \Gamma, n}^{\dagger}\right\rangle=\sum_{\hat{R}} \mid \psi_{\hat{R}}^{\dagger} \bar{S}_{R, \Gamma n}
$$


Correspondingly, pion-pion operators that project onto irreducible representations can be computed:

$$
\Psi_{i, \Gamma, n}^{\dagger}=\sum_{\hat{R}} \psi_{\hat{R}}^{\dagger} \bar{S}_{R, i \Gamma n}
$$

\subsection{Correlation Function}

Employing the operator $\psi_{R}$ at the source and sink yields the following two-point correlation function,

$$
G_{\hat{R}, \hat{R}^{\prime}}(t ; \vec{p} ; \vec{x}, \vec{\delta}) \equiv \sum_{(\vec{y}-\vec{x}) \in \mathbb{Z}^{3}} e^{i \vec{p} \cdot(\vec{y}-\vec{x})}\left\langle T\left(\psi_{\hat{R}}(t ; \vec{y}, \vec{\delta}), \psi_{\hat{R}^{\prime}}^{\dagger}(0 ; \vec{x}, \vec{\delta})\right)\right\rangle=G_{\hat{R}^{\prime-1} \hat{R}, \hat{I}}(t ; \vec{p} ; \vec{x}, \vec{\delta}),
$$

while the correlation function for the two pion operator $\Psi_{i, \Gamma, n}$ can be written as:

$$
\tilde{G}_{\Gamma n, \Gamma^{\prime} n^{\prime}}(t ; \vec{p} ; \vec{x}, \vec{\delta})=\sum_{(\vec{y}-\vec{x}) \in \mathbb{Z}^{3}} e^{i \vec{p} \cdot(\vec{y}-\vec{x})} \sum_{i}\left\langle T\left(\Psi_{i, \Gamma, n}(t ; \vec{y}, \vec{\delta}), \Psi_{i, \Gamma^{\prime}, n^{\prime}}^{\dagger}(0 ; \vec{x}, \vec{\delta})\right)\right\rangle
$$

Through Eq.(9) and the properties of group, we get:

$$
\tilde{G}_{\Gamma n, \Gamma^{\prime} n^{\prime}}(t ; \vec{p} ; \vec{x}, \vec{\delta})=\delta_{\Gamma, \Gamma^{\prime}} \delta_{n, n^{\prime}} \sum_{\hat{R}} \chi_{\hat{R}}^{\Gamma} G_{\hat{R}, \hat{I}}(t ; \vec{p} ; \vec{x}, \vec{\delta}) \equiv \tilde{G}_{\Gamma}(t ; \vec{p} ; \vec{x}, \vec{\delta}),
$$

where $\chi(\hat{R})$ is the character number of element $\hat{R}$ of the $O_{h}$ group in the irreducible representation $\Gamma$.

Our task is to calculate $G_{\hat{R}^{-1}, \hat{l}}(t ; \vec{p} ; \vec{x}, \vec{\delta})$ as follows:

$$
\begin{aligned}
& \left\langle G_{\hat{R}^{-1}, \hat{i}}(t ; \vec{p}=0 ; \vec{x}, \vec{\delta})\right\rangle \\
& =\sum_{(\vec{y}-\vec{x}) \in \mathbb{Z}^{3}}\left\{\operatorname{Tr}\left[S_{d}\left(\vec{y}^{-}, t ; \vec{x}^{-}\right) S_{u}^{\dagger}\left(\vec{y}^{-}, t ; \vec{x}^{-}, 0\right)\right] \operatorname{Tr}\left[S_{d}\left(\vec{y}^{+}, t ; \vec{x}^{+}, 0\right) S_{u}^{\dagger}\left(\vec{y}^{+}, t ; \vec{x}^{+}, 0\right)\right]\right. \\
& \quad+\operatorname{Tr}\left[S_{d}\left(\vec{y}^{+}, t ; \vec{x}^{-}, 0\right) S_{u}^{\dagger}\left(\vec{y}^{+}, t ; \vec{x}^{-}, 0\right)\right] \operatorname{Tr}\left[S_{d}\left(\vec{y}^{-}, t ; \vec{x}^{+}, 0\right) S_{u}^{\dagger}\left(\vec{y}^{-}, t ; \vec{x}^{+}, 0\right)\right] \\
& \quad-\operatorname{Tr}\left[S_{d}\left(\vec{y}^{-}, t ; \vec{x}^{-}, 0\right) S_{u}^{\dagger}\left(\vec{y}^{+}, t ; \vec{x}^{-}, 0\right) S_{d}\left(\vec{y}^{+}, t ; \vec{x}^{+}, 0\right) S_{u}^{\dagger}\left(\vec{y}^{-}, t ; \vec{x}^{+}, 0\right)\right] \\
& \left.\quad-\operatorname{Tr}\left[S_{d}\left(\vec{y}^{+}, t ; \vec{x}^{-}, 0\right) S_{u}^{\dagger}\left(\vec{y}^{-}, t ; \vec{x}^{-}\right) S_{d}\left(\vec{y}^{-}, t ; \vec{x}^{+}, 0\right) S_{u}^{\dagger}\left(\vec{y}^{+}, t ; \vec{x}^{+}, 0\right)\right]\right\} .
\end{aligned}
$$

Here, $S_{u}(\vec{y}, t ; \vec{x}, 0)=T(u(\vec{y}, t), \vec{u}(\vec{x}, 0))$ is quark operator, and we introduce the notation $\vec{x}^{ \pm}=\vec{x} \pm \vec{\delta} / 2$ and $\vec{y}^{ \pm}=\vec{y} \pm \hat{R} \vec{\delta} / 2$. Isospin symmetry $S_{u} \equiv S_{d}$ is assumed for the quark propagators. The four terms in Eq. (13) correspond to the Wick contractions as shown in Fig.1.

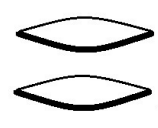

(a)

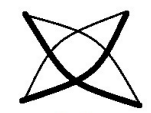

(b)

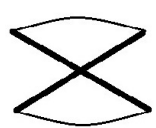

(C)

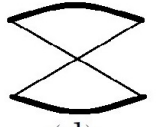

(d)

Figure 1. The diagrams for the Wick contractions of $\pi \pi \rightarrow \pi \pi$ with isospin-2. Thick and thin lines denote the $d \bar{d}$ and $u \bar{u}$ contractions, respectively.

From Eq.(10) or Eq.(12), two correlation functions can be calculated. However, the first one will have a very dense spectrum, overlapping (in principle) with all irreducible representations:

$$
G_{\hat{R}^{-1}, \hat{I}}(t ; \vec{p} ; \vec{x}, \vec{\delta}) \sim \sum_{i}\left\{Z_{i}^{A_{1}^{+}} e^{-E_{i}^{A_{1}^{+}} t}+Z_{i}^{A_{2}^{+}} e^{-E_{i}^{A_{2}^{+}} t}+Z_{i}^{E_{2}^{+}} e^{-E_{i}^{E^{+}} t}+Z_{i}^{T_{1}^{+}} e^{-E_{i}^{T_{1}^{+}} t}+Z_{i}^{T_{2}^{+}} e^{-E_{i}^{T_{2}^{+}} t}\right\},
$$


where the $\sum_{i}$ indicates a sum over all possible excited states within each irreducible representation. In fact, the ground-state energy of $A_{1}^{+}$is dominated by an $S$-wave state with zero momentum in the $\mathrm{CM}$ frame. It will therefore have the lowest energy and hence, in principle, the large Euclidean time behavior of this correlator will be saturated by this eigenstate. However, as shown in Eq.(12), it is possible to project directly onto the irreducible representation, $\Gamma$, and hence:

$$
\tilde{G}_{\Gamma}(t ; \vec{p} ; \vec{x}, \vec{\delta}) \sim \sum_{i} Z_{i}^{\Gamma} e^{-E_{i}^{\Gamma} t}
$$

In summary, we have shown how it is possible to extract the spectrum of each unique irreducible representation by constructing the appropriate sink-projected correlation function, $\tilde{G}$.

\subsection{Lüscher's equation for high partial waves}

In the $\pi \pi$ system with isospin-2, only positive parity and even angular momentum states are possible. In our following lattice simulation, we are able to extract the ground states of the $A_{1}^{+}, E^{+}$and $T_{2}^{+}$ representations. Therefore, we limit the angular momentum to $l \leq 4$. From Ref. [3], the formulae between partial wave phase shifts and the energy levels can be derived. In the case here, each irreducible representation has two partial waves up to $l=4$. Thus the eigenvalue equation will have a $2 \times 2$ matrix as follows:

$$
\operatorname{det}\left(\begin{array}{cc}
\cot \delta_{l_{1}}\left(q_{\Gamma}\right)+M_{11}^{\Gamma}\left(q_{\Gamma}\right) & M_{12}^{\Gamma}\left(q_{\Gamma}\right) \\
M_{12}^{\Gamma}\left(q_{\Gamma}\right) & \cot _{l_{2}}\left(q_{\Gamma}\right)+M_{22}^{A_{1}}\left(q_{\Gamma}\right)
\end{array}\right)=0,
$$

where

$$
M_{i j}^{\Gamma}(q)=\sum_{s} C_{i, j, s}^{\Gamma} \frac{Z_{s 0}\left(1 ; \frac{q L}{2 \pi}\right)}{\sqrt{(2 s+1) \pi^{3}}\left(\frac{q L}{2 \pi}\right)^{s+1}} .
$$

The indices $l_{1}, l_{2}$ indicate which two angular momenta are contained in the irreducible representation $\Gamma q(\Gamma)$ is the on-shell momentum of the energy level of irreducible representation $\Gamma$, while the function $Z_{s 0}$ is the zeta function defined in Ref. [3]. The indices $l_{1}, l_{2}, s$ and the coefficient $C_{i, j, s}^{\Gamma}$ are all listed in Table 3.

Table 3. The values of indexes $l_{1}, l_{2}, s$ and coefficients $C_{i, j, s}^{\Gamma}$ for each irreducible representation.

\begin{tabular}{llll}
\hline$\Gamma$ & $\left(l_{1}, l_{2}\right)$ & $(i, j)$ & $\left(s, C_{i, j, s}^{\Gamma}\right)$ \\
\hline$A_{1}^{+}$ & $(0,4)$ & $(1,1)$ & $(0,1)$ \\
& & $(1,2)$ & $\left(4, \frac{6 \sqrt{21}}{7}\right)$ \\
& & $(2,2)$ & $(0,1),\left(4, \frac{224}{14}\right),\left(6, \frac{80}{11}\right),\left(8, \frac{560}{143}\right)$ \\
$E^{+}$ & $(2,4)$ & $(1,1)$ & $(0,1),\left(4, \frac{18}{7}\right)$ \\
& & $(1,2)$ & $\left(4,-\frac{120 \sqrt{3}}{77}\right),\left(6,-\frac{30 \sqrt{3}}{11}\right)$ \\
& & $(2,2)$ & $\left(4, \frac{324}{1001}\right),\left(6,-\frac{64}{11}\right),\left(8, \frac{392}{143}\right)$ \\
$T_{2}^{+}$ & $(2,4)$ & $(1,1)$ & $(0,1),\left(4,-\frac{12}{7}\right)$ \\
& & $(1,2)$ & $\left(4,-\frac{60 \sqrt{3}}{77}\right),\left(6, \frac{40 \sqrt{3}}{11}\right)$ \\
& & $(2,2)$ & $(0,1),\left(4,-\frac{162}{77}\right),\left(6, \frac{20}{11}\right)$ \\
\hline
\end{tabular}




\section{Results}

\subsection{Numerical calculation}

As it stands, Eq. (12) implies calculating $48 G_{\hat{R}, \hat{I}}$ correlation functions corresponding to the different rotations $\hat{R}$. However, for each measurement, we only require two sources for the quark propagators at positions at $\vec{x} \pm \vec{\delta} / 2$. The 48 rotations can be summed over at the sink of the correlation function. This significantly reduces the computational overhead of additional propagator inversions.

The present calculation is performed on an ensemble with 2 flavours of dynamical $O(a)$ improved Wilson fermions with $(\beta, \kappa, V)=\left(5.29,0.13550,24^{3} \times 48\right)$, corresponding to $\left(a, m_{\pi}=\right.$ $(-0.071 \mathrm{fm}, 900 \mathrm{MeV})$ [13]. Results are collected from 376 configurations using 16 different source locations, with 2 inversions for each compound source. In Fig. 2, the plateau of $\log \left(\tilde{G}_{\Gamma}(t) / \tilde{G}_{\Gamma}(t+1)\right)$ are identified in the $A_{1}^{+}, E^{+}$and $T_{2}^{+}$representations. By choosing suitable fitting windows, the energy levels are determined. Our (preliminary) results for the corresponding on-shell momenta are: $\frac{p_{A_{1} L}}{2 \pi}=0.2455(36), \frac{p_{E} L}{2 \pi}=1.047(13)$, and $\frac{p_{T_{2}} L}{2 \pi}=1.457(23)$. As shown in Ref. [7, 11], the lowest momentum the non-interacting two-pion state in the $A_{1}^{+}$can have is $|\vec{p} L / 2 \pi|=0$, while for $E$ it is $|\vec{p} L / 2 \pi|=1$ and $T_{2}$ is $|\vec{p} L / 2 \pi|=\sqrt{2}$. Our determined momenta appear to indicate a weak repulsion in each of these channels. It is certainly encouraging that the ground-state energies in each of the irreducible representations are being reliably determined.

By using this method, the momentum of each pion has not been specified. Only the total momentum of the system is input - for which we have only considered $\vec{P}=\overrightarrow{0}$ in this study. In contrast to formulations that involve a momentum-projected hadron at the sink [9, 12], we use the same operator at both source and sink. This method then lends itself to a computationally-efficient technique for extension to a variational analysis [14], where the operator basis can be extended by varying $\delta$.

\subsection{Phase shifts}

Equation (16) defines a relationship between phase shifts and the finite-volume energy levels. Since we only have one eigenvalue in each irreducible representation, at three different on-shell momenta, we cannot solve for the phase shifts directly. For illustration, we parameterise the phase shifts in terms of an effective range expansion as follows [11, 15-18]:

$$
q^{2 l+1} \delta_{l}=-\frac{1}{a_{l}}+\frac{1}{2} r_{l} q^{2}
$$

Since the S-wave only appears in the $A_{1}^{+}$irreducible representation, we can solve for this phase shift directly. With the present calculation, the best we can do is then extract the scattering length for $l=2,4$, neglecting the $r_{l}$ terms. In Fig. 3, we show the phase shifts based on the determination of the scattering lengths. A strong signal for $\delta_{2}$ is observed. The determination of $\delta_{4}$ is clearly pushing the limits of our statistical precision. Clearly we do not anticipate attraction in this channel, yet it is encouraging that we are on the cusp of producing a meaningful signal for $l=4$. Obviously, extending to a variational analysis to gain access to more excited states, and including boosted states for a more dense spectrum [11] will dramatically improve the available constraints.

\section{Summary}

In this paper, we introduce a new extended operator to extract the spectra of irreducible representations. In coordinate space, the two-particle operator projects onto an irreducible representation by summing appropriately over a spherical shell. The method is computationally effecient, as it only 


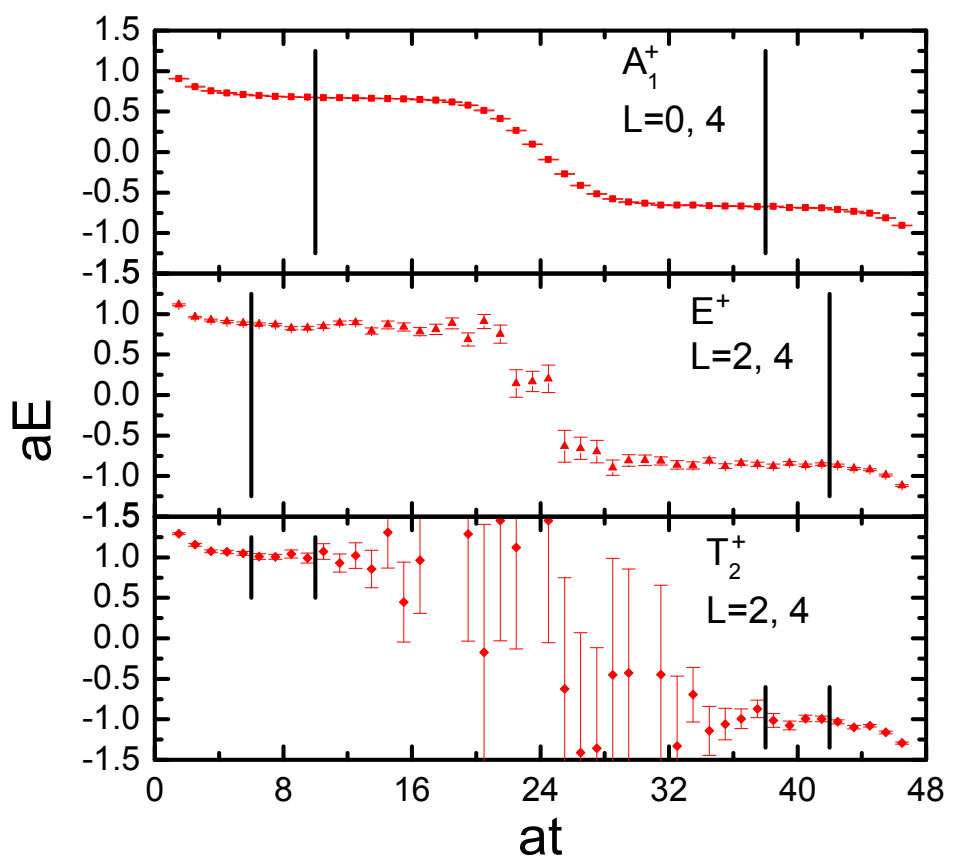

Figure 2. The effective energy $a E$ of $A_{1}^{+}, E^{+}$and $T_{2}^{+}$representations for the rest frame on a $24^{3} \times 48$ lattice with $a=0.071 \mathrm{fm}$ and $m_{\pi} \approx 900 \mathrm{MeV}$. The thick black lines show the limits of the fitting windows.

requires two source inversions per measurement. Having the same operator as source and sink will allow a straightforward implementation of a variational analysis.

For the numerical investigation in this work, we studied the isospin- $2 \pi \pi$ system in the CM frame on a $24^{3} \times 48$ lattice with a lattice spacing of $a=0.071 \mathrm{fm}$ and $m_{\pi} \approx 900 \mathrm{MeV}$. The spectra of $A_{1}^{+}$, $E^{+}$and $T_{2}^{+}$irreducible representations of a $\pi^{-} \pi^{-}$system are successfully extracted. The phase shifts can be calculated through the Lüscher equation with a toy model parameterisation. In the future, this method can also be readily extended to boosted frames, where the rotational symmetries on the lattice are reduced to the little groups.

\section{Acknowledgements}

The simulations were undertaken using supercomputing resources provided by the Phoenix HPC service at the University of Adelaide and at the NCI National Facility in Canberra, Australia. The NCI resources are provided through the National Computational Merit Allocation Scheme and the University of Adelaide Partner Share supported by the Australian Government. We have employed the Chroma software library [19] in our analysis. This investigation has been supported by the Australian Research Council under grants FT120100821, FT100100005 and DP140103067. GS was supported by DFG grant SCHI 179/8-1. 


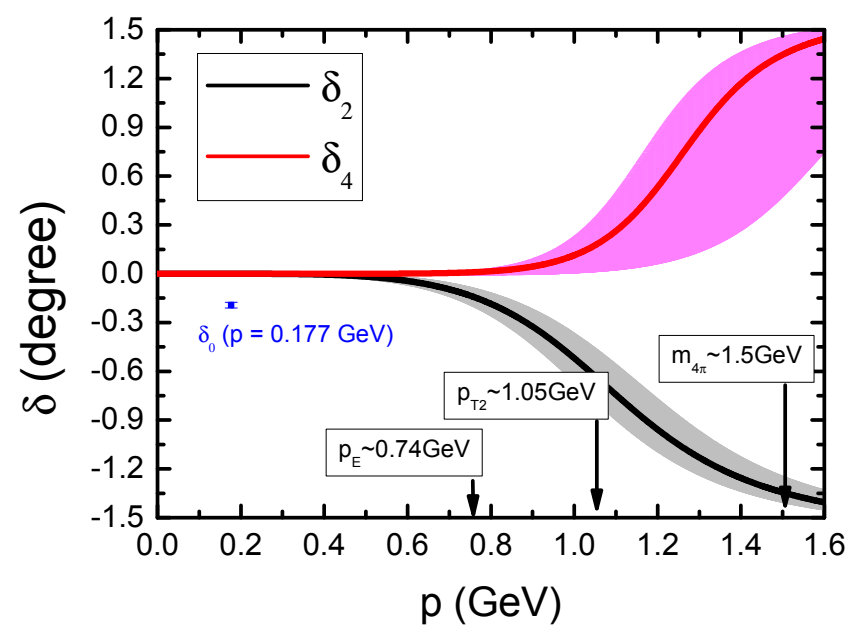

Figure 3. The fitting result from the spectra in the rest frame with lattice size $L=24$ and spacing $a=0.071 \mathrm{fm}$. The two parameters $a_{2}$ and $a_{4}$ are $-0.571 \pm 0.155 \mathrm{GeV}^{-5}$ and $0.114 \pm 0.100 \mathrm{GeV}^{-9}$, respectively. The value for $\delta_{0}(p=0.177 \mathrm{GeV})=-0.190 \pm 0.017$.

\section{References}

[1] C. Liu, PoS LATTICE2016, 006 (2017), 1612.00103

[2] R.A. Briceno, J.J. Dudek, R.D. Young (2017), 1706. 06223

[3] M. Lüscher, Nucl. Phys. B354, 531 (1991)

[4] M. Lüscher, Commun. Math. Phys. 105, 153 (1986)

[5] V. Bernard, M. Lage, U.G. Meissner, A. Rusetsky, JHEP 08, 024 (2008), 0806.4495

[6] M. Göckeler et al., Phys. Rev. D86, 094513 (2012), 1206.4141

[7] T. Luu, M.J. Savage, Phys. Rev. D83, 114508 (2011), 1101. 3347

[8] J.J. Wu, T.S.H. Lee, D.B. Leinweber, A.W. Thomas, R.D. Young, JPS Conf. Proc. 10, 062002 (2016), 1512.02771

[9] E. Berkowitz et al., Phys. Lett. B765, 285 (2017), 1508.00886

[10] J.J. Dudek et al., Phys. Rev. D83, 071504 (2011), 1011.6352

[11] J.J. Dudek, R.G. Edwards, C.E. Thomas, Phys. Rev. D86, 034031 (2012), 1203.6041

[12] S.R. Beane et al. (NPLQCD), Phys. Rev. D85, 034505 (2012), 1107.5023

[13] G.S. Bali et al., Nucl. Phys. B866, 1 (2013), 1206.7034

[14] B. Blossier et al., JHEP 04, 094 (2009), 0902 . 1265

[15] W. Hoogland et al., Nucl. Phys. B126, 109 (1977)

[16] D.H. Cohen, T. Ferbel, P. Slattery, B. Werner, Phys. Rev. D7, 661 (1973)

[17] A. Zieminski et al., Nucl. Phys. B69, 502 (1974)

[18] N.B. Durusoy et al., Phys. Lett. 45B, 517 (1973)

[19] R.G. Edwards, B. Joo, Nucl. Phys. Proc. Suppl. 140, 832 (2005), hep-lat/0409003 